\title{
Tear Film Biomarkers in Dry Eye Disease
}

\author{
Mohamed Hantera \\ Umm Al-Qura University, Makkah, Saudi Arabia; Medical Reference Center, Jeddah, Saudi Arabia
}

DOI: https://doi.org/10.17925/USOR.2020.13.2.68

$\mathrm{D}$ iagnosis of dry eye disease is challenging due to poor correlation between clinical signs and symptoms. The most commonly reported diagnostic tools used by practitioners (patient symptoms, tear break-up time, and vital staining of the ocular surface) are not always sensitive enough to detect mild disease and effectively monitor therapeutic response. The proximal position of the tear film to the ocular surface, and the relative ease of sampling, could make tear film analysis a promising tool for the diagnosis and management of dry eye disease. This review investigates the current research into tear film biomarkers and their potential use for diagnosis and treatment of the disease. Tear film biomarkers are evolving as a more sensitive measure than other functional tools. They have also been found to be better markers of disease severity than corneal staining and Schirmer's test. Current research confirms that the diagnosis of dry eye disease is still based on symptoms and clinical presentation, but this could be combined with biomarker analysis to formulate a more accurate diagnosis and targeted therapy. Future research should be focused on developing a robust tool for tear fluid sampling that is efficient and cost-effective in a clinical setting.

\section{Keywords}

Evaporation, aqueous deficiency, tear film biomarkers, osmolarity, dry eye disease, tear film stability

Disclosure: Mohamed Hantera has no financial or non-financial relationships or activities to declare in relation to this article.

Acknowledgments: Writing and editing assistance was provided by Louise Wood, Eye Write Communications.

Review Process: Double-blind peer review.

Compliance with Ethics: This study involves a review of the literature and did not involve any studies with human or animal subjects performed by the author.

Authorship: The named author meets the International Committee of Medical Journal Editors (ICMJE) criteria for authorship of this manuscript, takes responsibility for the integrity of the work as a whole, and has given final approval for the version to be published.

Access: This article is freely accessible at touchOPHTHALMOLOGY.com (C) Touch Medical Media 2021

Received: November 15, 2020

Accepted: December 10, 2020

Published Online: January 27, 2021

Citation: US Ophthalmic Review. 2020;13(2):68-72

Corresponding Author: Mohamed Hantera, Medical Reference Center, King Road, Jeddah, KSA.

E: Mohamedhantera30@gmail.com

Support: No funding was received in the publication of this manuscript.
Dry eye disease is a chronic condition that affects the corneal surface, and is clinically characterized by loss of tear volume and increased evaporation of tears., ${ }^{1,2}$ The definition of dry eye disease has evolved since it was first recognized over 30 years ago, with advanced research resulting in a better understanding of the disease pathophysiology. ${ }^{3}$ The official report in 2017 from the Tear Film and Ocular Surface Society (TFOS) International Dry Eye Workshop II (DEWS II) defined dry eye disease as "a multifactorial disease of the ocular surface characterized by a loss of homeostasis of the tear film, and accompanied by ocular symptoms, in which tear film instability and hyperosmolarity, ocular surface inflammation and damage, and neurosensory abnormalities play etiological roles. ${ }^{13}$ The report classifies dry eye disease into two main subtypes: evaporative dry eye and aqueous-deficient dry eye disease, and describes a band of overlap between these sub-categories. ${ }^{3}$ A number of factors can disrupt tear film homeostasis: eyelid and blink abnormalities, ocular surface irregularities, tear component deficiencies, and neurosensory abnormalities. ${ }^{1}$ Ocular health is largely influenced by a stable healthy tear film. ${ }^{1}$ The composition of the tear film affects the refractive optics of the eye and provides moisture to the ocular surface. ${ }^{1}$

With dry eye disease, there is not always a direct correlation between symptoms and signs, which leads to misdiagnosis. ${ }^{3}$ Despite the TFOS DEWS II guidelines indicating tear film homeostasis as a key component in dry eye disease, the most frequently used tools for diagnosis among practitioners remain patient symptoms and a combination of tear break-up time (TBUT) and ocular surface stain. ${ }^{4-12}$

Treatment for dry eye disease is dependent on disease severity. The 2017 TFOS DEWS II provides guidelines for a staged management and treatment protocol for dry eye disease. ${ }^{13}$ Recent practitioner survey results confirm mild dry eye is being treated with non-preserved artificial tear substitutes combined with lid hygiene.,4,-9,11 The addition of non-preserved gels or ointments and topical anti-inflammatories is the mainstay for treatment of moderate to severe dry eye. ${ }^{4,79,11}$ Efficacy of treatment is assessed by an improvement in patient-reported symptoms and a reduction in ocular signs. The identification of new tools focusing on tear film biomarker assessment could improve therapeutic efficacy.

This review aims to investigate the advantages of tear film biomarker analysis in dry eye disease diagnosis and management, and to determine if it is a widely used tool among practitioners. A literature search using PubMed was conducted using keywords to determine the available research into tear film biomarkers for evaluating dry eye disease. This is a newly evolving topic that may change the way we diagnose and manage dry eye disease. The current diagnostic tools for dry eye disease could be enhanced by tear film biomarker detection and understanding. 


\section{The tear film}

The tear film provides lubrication to maintain the health and homeostasis of the ocular surface, thereby preserving the optical clarity of the eye. ${ }^{14}$ The three-layer physiology of the tear film, first described last century by Wolff, is still widely utilized: a mucin layer covering the epithelial cells of the cornea, an aqueous layer to provide lubrication and nutrients to maintain osmolarity, and a lipid layer to prevent evaporation. ${ }^{1,15}$ Doane, more than 20 years ago, labelled this model as "only useful as an approximation" and as "a considerable simplification." ${ }^{16}$ He described the oily lipid portion of the tear film as a thin layer on the anterior surface, consistent with Wolff's proposal, but suggested the other components may not be as easily stratified. ${ }^{16}$

The TFOS DEWS II described the precorneal tear film as behaving as a "single dynamic functional unit with different compartments." ${ }^{\prime 3}$ The aqueous and mucin layers are described as a single layer of muco-aqueous gel, and the lipid layer is secreted by the meibum on the lid margins and plays an important role in stabilizing the tear film. ${ }^{1}$ The lipid layer thickness can be measured by analyzing the color and brightness of interference images. ${ }^{1}$ It has a mean thickness of $42 \mathrm{~nm}(15-157 \mathrm{~nm}) .{ }^{17}$ The lipid layer is rich in polar lipids and some long-chain fatty acids with a superficial layer of non-polar lipids. ${ }^{16}$ Some proteins and glycoproteins are presumed to be distributed within the lipid layer to enhance its stability. ${ }^{17}$

The components of the muco-aqueous layer can be variable, and the knowledge of distribution is still evolving.' The muco-aqueous layer is freshly deposited with each blink and contains goblet cell mucin and proteins produced by the lacrimal gland, conjunctiva, and meibomian gland. ${ }^{17}$

Tear film osmolarity is now an established factor in the pathogenesis of both aqueous-deficient dry eye and evaporative dry eye. Evaporation in between blinks causes a thinning of the tear film and a rise in tear osmolarity. ${ }^{17}$ Local areas of hyperosmolarity within the tear film are considered to be a source of repeated stress to the ocular surface. ${ }^{17}$ The TFOS DEWS II report describes the tear film lipid layer as being instrumental in the rate of evaporation from the eye. ${ }^{17}$ When the lipid layer is lacking (measured with interferometry), evaporation increases, contributing to a corresponding increase in tear osmolarity. ${ }^{17}$

\section{Dry eye disease pathophysiology}

The 2017 TFOS DEWS I| report stated that tear instability and tear hyperosmolarity were the two main factors involved in dry eye disease. ${ }^{17}$ This led to the classification of evaporative dry eye disease, the result of excessive evaporation form the tear film (with normal lacrimal function), and aqueous-deficient dry eye, the result of reduced lacrimal secretion with normal tear evaporation rates. ${ }^{17}$ Meibomian gland dysfunction, resulting in a reduction in the tear film lipid layer, is the typical cause of evaporative dry eye, whilst aqueous-deficient dry eye can be related to lacrimal gland excretion deficiency. ${ }^{17}$ In any form of dry eye disease, once tear break-up is observed between a blink cycle, then an additional evaporative element is initiated. ${ }^{17}$ Environmental factors can contribute to the evaporative state and result in hyperosmolarity; for example atmospheric humidity, wind, blink rate, and systemic medications. This tear film hyperosmolarity, no matter the duration, will initiate an inflammatory response, which, if left untreated, can perpetuate a vicious cycle of dry eye disease. ${ }^{18}$

\section{Dry eye disease diagnosis}

\section{Functional and subjective tests}

Current research encourages a tear-film oriented diagnosis that looks at the tear secretion volume, tear stability, lipid layer thickness, and vital staining to evaluate dry eye disease. ${ }^{19}$ The Japanese Dry Eye Society and Asia Dry Eye Society base their dry eye disease diagnosis on this concept. ${ }^{19}$

Although the use of multiple tests can determine the multifactorial etiology of dry eye disease, the consensus among eye care practitioners is that tear osmolarity is best used as a disease severity marker. ${ }^{20-22}$ Using the tear-film oriented diagnosis approach, a range of clinical examinations can be used to ascertain the reason for the clinical signs and symptoms. ${ }^{19}$ The aqueous tear film layer quantity can be evaluated using the Schirmer test, the strip meniscometry, or anterior segment optical coherence tomography. ${ }^{19}$ The Schirmer test may be useful to assess tear secretion, but it is invasive and has limited reproducibility. ${ }^{19}$ Lipid interferometry is available to evaluate the lipid layer. ${ }^{19}$ Clinically evaluating each tear film abnormality can lead to a targeted and more efficient treatment approach. ${ }^{19}$ Despite the consensus among practitioners that tear osmolarity is an important factor, practitioner survey results from several recent papers listed patient symptoms, a combination of TBUT, meibomian gland evaluation, and ocular surface stain as the predominant clinical tools still being used to diagnose dry eye. .-12,23 $^{-12}$

\section{Tear film biomarkers}

Research is now focused on molecular biomarkers indicative of dry eye disease. It is proposed that this may give a more quantitative measure of disease severity and therapeutic effectivity. The tear film fluid is proximal to the ocular surface, so evaluating its composition and detecting reliable biomarkers for ocular surface disease could enhance diagnosis and management. ${ }^{14}$

Despite multiple qualitative and quantitative measures to evaluate the ocular surface and tear film, there is no consensus as to what tests should be routinely utilized. ${ }^{24}$ In addition, with the vast spectrum of severity in dry eye disease, there are no definitive threshold values to diagnose and manage treatment effectiveness. ${ }^{24}$ Often, the sampling tools used can affect the diagnosis severity by the administration of drops and reflex tear secretions. ${ }^{24}$

A tear film biomarker is measured objectively and can indicate the biological responses in the body. ${ }^{25}$ Tear film sampling is relatively non-invasive, and biomarker investigation could lead to a more selective and accurate disease prediction. ${ }^{25}$ A biomarker, defined by the National Institutes of Health, is "a characteristic that is objectively measured and evaluated as an indicator of normal biological processes, pathogenic processes, or pharmacologic responses to therapeutic intervention." ${ }^{26}$

The use of tear film biomarkers as a tool for dry eye disease diagnosis and management is evolving in the literature due to frequent discordance between clinical signs and symptoms. ${ }^{27}$ Roy et al. describes an ideal biomarker as having relevance to the clinical setting and disease severity, being easy and economical to use, reproducible, and having undergone multiple clinical trials establishing any bias. ${ }^{24}$ 
Table 1: Inflammatory tear film biomarkers ${ }^{24,28}$

\begin{tabular}{|l|l|}
\hline Inflammatory biomarker & Value as a biomarker \\
\hline TNF- $\alpha$ & General inflammatory status of the ocular surface \\
\hline IL-6 & Pro- and anti-inflammatory roles \\
\hline IL-17A & Secreted by specialized T helper 17 subpopulation \\
\hline IL-8 & $\begin{array}{l}\text { Important in chemotaxis to mediate macrophage and } \\
\text { epithelial innate immunity }\end{array}$ \\
\hline MMP-9 & $\begin{array}{l}\text { Lack of correlation with other clinical tests and } \\
\text { symptoms }\end{array}$ \\
\hline
\end{tabular}

$I L=$ interleukin; $M M P=$ matrix metalloproteinases; $T N F=$ tumor necrosis factor.

\section{Inflammatory biomarkers}

When inflammation was discovered to be one of the important features of dry eye disease, research established inflammatory mediators in tears to be key indicators for ocular surface inflammation. ${ }^{24,28}$ These biomarkers are not specific to dry eye disease, as other ocular surface disorders, such as allergy and infection, also cause inflammation. Inflammation in dry eye disease involves both innate and adaptive immune responses; an increase in tear film osmolarity initiates an innate immune response. ${ }^{29}$ The immune homeostasis of the ocular surface is controlled by lymphocytes and regulatory $T$ cells, ${ }^{29}$ these interact with anti-inflammatory factors like interleukin (IL)-1, transforming growth factor (TGF)- $\beta 2$, and matrix protease inhibitors. ${ }^{29}$ The most frequently reported inflammatory biomarkers linked to dry eye disease in the current literature are cytokines and chemokines, tumor necrosis factor-alpha (TNF- $\alpha$ ), IL-17A, IL-6, IL-8, and matrix metalloproteinase9 (MMP-9) (Table 1)..$^{24,28}$

Despite the presence of these biomarkers in the tear film, the variation in concentration in healthy subjects is significant, and for this reason comparing percentage change in an individual may be a more valuable way to determine treatment effectiveness. ${ }^{24}$ Minimal differences have been found between levels of cytokines and chemokines in those with mild dry eye disease and healthy subjects. ${ }^{28}$ Further studies are required to ascertain any reliable association with disease severity and response to treatment. A simple method for clinical sampling and analysis is also required to make use of this diagnostic tool.

Components of lipid metabolism have also been reported to correlate with clinical measures of dry eye disease, these include secretory phospholipase A2, prostaglandin E2, arachidonic acid, docosahexaenoic acid, eicosapentaenoic acid, and leukotriene B4..$^{24}$ In addition, MMP-9 is an enzyme that contributes to tissue remodeling in inflammation. ${ }^{24}$ Several studies have shown elevated levels of MMP-9 in the tear film of patients with dry eye disease. ${ }^{24}$ However, this elevation has also been associated with other ocular surface diseases, such as ocular allergy, ocular rosacea, and acanthamoeba/herpetic keratitis. ${ }^{24,30}$ The levels often do not correlate well with disease symptoms and other clinical signs. ${ }^{24}$ MMP-9 expression in dry eye disease represents ocular tissue damage, but cannot be used as a diagnostic tool; however, disease severity and therapeutic effectiveness may be monitored by measuring MMP-9 levels in the tear film..$^{30}$ InflammaDry ${ }^{\circledR}$ (Quidel, San Diego, CA, USA) is a commercially available tool to measure the levels of MMP-9 in the tear film..$^{30}$

There is extensive research into specific proteins and enzymes found in tear samples that are associated with ocular diseases, such as
Table 2: Important tear film biomarkers in dry eye disease ${ }^{27,30,32}$

\begin{tabular}{|l|l|}
\hline Classification & Tear film biomarkers \\
\hline Inflammatory & Lactoferrin, albumin, cytokines/chemokines, MMP-9 \\
\hline Lacrimal & Lactoferrin, Iysozyme, growth factors \\
\hline Oxidative stress & Lactoferrin, lipid peroxidation, antioxidant defense \\
\hline Tear film osmolarity & $\begin{array}{l}\text { Variation in measurements across normal subjects and } \\
\text { no consistent thresholds }\end{array}$ \\
\hline
\end{tabular}

MMP-9 = matrix metalloproteinase-9.

Stevens-Johnson syndrome, Sjogren's Syndrome, and others. ${ }^{25}$ Some of these diseases exhibit tear film biomarkers that overlap with dry eye disease. A thorough clinical history will help distinguish dry eye disease from other ocular surface diseases in the presence of a common inflammatory biomarker.

\section{Dry eye disease biomarkers}

There is a range of dry eye disease biomarkers currently reported in the literature (Table 2). ${ }^{28,30,32}$ These biomarkers have also been linked to other ocular diseases. ${ }^{28,30}$ There are two commonly accepted biomarkers for dry eye disease: lactoferrin and lysozyme. ${ }^{30}$ Lactoferrin is a singlechain polypeptide that contains antimicrobial, antiviral, anti-inflammatory, and antioxidant properties. ${ }^{30}$ It has been found to promote healing of corneal epithelial cells in culture. ${ }^{1}$ A lactoferrin supplement was found to increase tearing when it was trialed in a randomized double-blind, placebo-controlled study in humans. ${ }^{1}$ It also restored tearing and TBUT in a randomized controlled study of patients with dry eye following cataract surgery. ${ }^{1}$ Lactoferrin and lysozyme are produced by the lacrimal gland, and a decrease in their levels can be due to lacrimal gland dysfunction, inflammation, and ocular surface damage. ${ }^{30}$ For a dry eye disease diagnosis, a threshold level of $1.1 \mathrm{mg} / \mathrm{mL}$ of tear lactoferrin has been found to have high sensitivity (79.4\%) and specificity (78.3\%).1.30 Currently, there is one commercial product that has been designed to investigate the tear film lactoferrin biomarker: TearScan ${ }^{\text {TM }}$ Lactoferrin Diagnostic Test Kit (Advanced Tear Diagnostics, Birmingham, AL, USA).

Lysozyme is a protein with antimicrobial properties. ${ }^{30}$ Lysozyme values in normal tears range from $1-3 \mathrm{mg} / \mathrm{mL}$, but can decrease with age; therefore, age-matched populations should be considered when assessing normal levels. ${ }^{31}$ In 2010, Versura et al. confirmed that a decrease in lactoferrin and lysozyme, and an increase in albumin can indicate an early inflammatory reaction in dry eye disease, which can be detected before clinical signs. ${ }^{32}$ Levels of lactoferrin of $<18 \%$, lysozyme of $<35 \%$, and albumin of $>15 \%$ have been reported as unique for severe dry eye disease. ${ }^{30}$

Tear proteomic analysis has recognized more than 500 proteins as biomarkers for dry eye disease. ${ }^{28,30,32}$ Multiple studies have found deficiency of Iysozyme-C, lipocalin 1, lactoferrin, Iysozyme proline-rich protein 4 and 3 , in addition to other proteins in dry eye disease. ${ }^{14}$ Current research supports increased levels of annexin 2, enolase $1 \alpha$, albumin, nerve growth factor, clusterin, $\beta 2$ microglobulin, calgranulin A (S100 A8) and B (S100 A9), cystatin SN, cathepsin S, defensins $\alpha$ and $\beta$, glycoprotein 340, and secretoglobin 2A2; and decreased levels of lactoferrin, lysozyme, lipocalin-1, annexin 5, alpha 2-glycoprotein 1, lacritin, caspase 14, proline-rich protein 3 and 4, cystatin S, cathepsin B, secretoglobin 1D1, prolactin inducible protein, and Mucin $5 \mathrm{AC} .{ }^{27,30,33}$ Despite the range 
of tear biomarkers that have been found using proteomic analysis, it is still not considered a useful diagnostic tool in clinical practice owing to the limited availability for analysis. ${ }^{30}$

Oxidative stress can also cause damage to the ocular surface. Environmental factors, preservatives in eyedrops, inflammatory reactions, and a decrease of lactoferrin and lysozyme contribute to oxidative stress. ${ }^{30}$ Lipid peroxidation and antioxidant defense markers have been elevated in patients with dry eye disease..$^{30}$ These biomarkers may be used for diagnosis but they will not necessarily distinguish between aqueous-deficient dry eye and evaporative dry eye disease..$^{30}$

Tear osmolarity has been reported in the literature as a potential dry eye disease biomarker. Measurement of tear osmolarity is relatively straightforward and small tear volumes are required; however, there seems to be a variation among studies, and the cut-off for hyperosmolarity is not clear. ${ }^{24}$ In addition, the variation in measurements for normal subjects in one session can be high. ${ }^{24}$ The TearLab osmolarity test (TearLab, Escondido, CA, USA) is a device used to measure osmolarity. A range of osmolarity of $>308 \mathrm{mOsm} / \mathrm{L}$ is used as a cut-off for diagnosis. ${ }^{14}$ The variability of the tear hyperosmolarity reduces the accuracy of the results. Suzuki et al. found tear osmolarity correlated with severity grading for dry eye disease. ${ }^{34}$ They found that less tear secretion associated with higher tear osmolarity in dry eye disease is consistent with increased evaporation rate. ${ }^{34}$

\section{Sampling for tear film biomarkers}

Currently, the collection of tear film biomarkers is uncommon in clinical practice. ${ }^{25}$ They are costly to analyze and there are significant variations in collection techniques which can influence the quality and quantity of the sample. ${ }^{25}$ Reflex tearing during sampling may also affect the concentration of proteins in the samples. ${ }^{25}$ For this reason, disease symptoms and clinical signs do not often correlate with biomarker levels.

Current methods for tear sampling include collection with Schirmer strips, sponges in the conjunctival meniscus, and micropipettes or glass capillaries at the outer conjunctival canthus..$^{30}$ As previously mentioned, there can be reflex tearing that affects sampling; however, results from the TFOS DEWS II report indicate no appreciable change in lactoferrin, lipocalin 1, and lysozyme in a closed eye or in basal and reflex tears. ${ }^{3}$ Analysis of tear proteins using the Hyrys-Hydrasys System (Sebia Inc, Norcross, GA, USA) also corroborated this finding, demonstrating that the electrophoresis pattern is not affected by reflex tears. ${ }^{30}$ Electrophoresis can be used for analysis of tear fluid and may be helpful in early diagnosis and management of dry eye disease.

The retention of some proteins (e.g. albumin and lysozyme) with Schirmer strips limits their use for tear protein analysis, and for this reason they are only recommended for the analysis of cytokines.,30 Multiplex immunoassays are costly, limiting tear film biomarker sampling in the clinical setting. In addition, the use of different sampling methods and different settings for the collection of the tears may increase the variability of the samples. Due to the variability in testing and the poor reproducibility, it has been suggested that reporting a percentage change in concentration rather than the absolute concentration of a biomarker may be a more accurate measure of disease severity and effective therapy. ${ }^{124,25}$

Despite the above limitations, the use of tear film biomarkers for dry eye disease diagnosis and management remains an area for future research and development. A minimally invasive measure to sample tear film and determine validated biomarkers with repeatability and accuracy is required. ${ }^{24}$ The results need to be proven to be reproducible across multiple studies. ${ }^{24}$ In-office testing is available for some biomarkers, but further development is required to gain a better understanding of each biomarker for accurate disease diagnosis and management. ${ }^{24}$

\section{Conclusion}

Current clinical trends in dry eye disease diagnosis and management rely on subjective and functional tools. Often, there is a poor correlation between dry eye disease clinical signs, symptoms, and severity, and these tools do not always accurately reflect the extent of the disease. With extensive research into tear film biomarkers, it is hoped that more accurate diagnosis of disease severity, and therefore management, will evolve; however, a current limiting factor is the difficulty in obtaining tear samples. ${ }^{25}$ In addition, further investigation is needed to form a better understanding of the disease process and the correlation of particular cytokines with clinical signs. ${ }^{25}$

Future research should be targeted at developing a robust tool for tear fluid sampling that is efficient in a clinical setting. Studies should be clear about where the tears are collected, and the type of tears (reflex, basal, or after sleep) analyzed. Sampling tears from the lower tear film meniscus does not necessarily reflect the osmolarity of the rest of the ocular surface. ${ }^{1}$ Techniques designed to measure the osmolarity over the cornea may give better insight into the diagnosis and treatments for dry eye disease. ${ }^{1}$ This may also be the case for tear film biomarker detection. Interest in tear film biomarkers for dry eye disease is increasing. A reduction in lactoferrin, lysozyme, growth factors, and mucins in the tears, as well as elevated levels of albumin, cytokines, chemokines and MMP-9 are emerging as the principal biomarkers for dry eye disease. ${ }^{24}$

Diagnosis of dry eye disease is still based on symptoms and clinical presentation, but in future this could be combined with biomarker analysis to formulate a more accurate diagnosis and targeted therapy. ${ }^{1}$ Despite the multitude of tear biomarkers for dry eye disease to date, not one has an absolute clinical value, and accurate diagnosis still relies on clinical history and signs alongside biomarker levels. ${ }^{1}$ Careful clinical history is also required to differentiate dry eye disease from other ocular surface disorders with common tear film biomarkers.

It is hoped that future research will provide us with a more detailed model of the tear film structure and function in dry eye disease, which will, in turn, allow a more targeted therapeutic plan. The need for minimally invasive and cost-effective methods to sample tear film in a clinical setting to identify and validate biomarkers of dry eye disease remains fundamental if we are to build our understanding of the mechanisms and treatment of dry eye disease. $\square$ 
1. Willcox MDP, Argüeso P, Georgiev GA, et al. TFOS DEWS II Tear Film Report. Ocul Surf. 2017;15:366-403

2. Rouen PA, White ML. Dry eye disease, prevalence, assessment and management. Home Healthc Now. 2018;36:74-83.

3. Craig JP, Nichols KK, Akpek EK, et al. TFOS DEWS II Definition and Classification Report. Ocul Surf. 2017:15:276-83.

4. Kim WJ, Kim HS, Kim MS. Status of diagnosis and treatment of patients with dry eye in Korea by survey. J Korean Ophthal. 2007:48:1614-22

5. Carona G, Serés C, Quevedo $L$, et al. Knowledge and use of tear film evaluation tests by Spanish practitioners. Optom Vis Sci. 2011;88:1106-11.

6. Echavez M, Lim Bon Siong R. Survey on the knowledge, attitudes, and practice patterns of ophthalmologists in the Philippines on the diagnosis and management of dry eye disease. Philipp J Ophthalmol. 2019;44:68-74.

7. Downie LE, Keller PR, Vingrys AJ. An evidence-based analysis of Australian optometrists' dry eye practices. Optom Vis Sci. 2013;90:1385-95

8. Williamson JF, Huynh K, Weaver MA, Davis RM. Perceptions of dry eye disease management in current clinical practice. Eye Contact Lens. 2014:40:111-5.

9. Downie LE, Rumney N, Gad A, et al. Comparing self-reported optometric dry eye clinical practices in Australia and the United Kingdom: is there scope for practice improvement? Ophthalmic Physiol Opt. 2016;36:140-51.

10. Asiedu K, Kyei S, Ayobi B, et al. Survey of eye practitioners' preference of diagnostic tests and treatment modalities for dry eye in Ghana. Cont Lens Anterior Eye. 2016;39:411-5.

11. Xue AL, Downie LE, Ormonde SE, Craig JP. A comparison of the self-reported dry eye practices of New Zealand optometrists and ophthalmologists. Ophthalmic Physiol Opt. 2017;37:191-201.

12. Bunya VY, Fernandez $K B$, Ying $G S$, et al. A survey of ophthalmologists regarding practice patterns for dry eye and Sjogren's syndrome. Eye Contact Lens. 2018;44:196-201.

13. Jones L, Downie L, Korb D, et al. TFOS DEWS II Management and Therapy Report. Ocul Surf. 2017;15:575-628.

14. Tamhane M, Cabrera-Ghayouri S, Abelian G, Viswanath V. Review of biomarkers in ocular matrices: challenges and opportunities Pharm Res. 2019;36:40.

15. Wolff $\mathrm{E}$. The Anatomy of the Eye and Orbit. 4th edn. London: HK Lewis and Co., 1954

16. Doane MG. Abnormalities of the structure of the superficial lipid layer on the in vivo dry-eye tear film. Adv Exp Med Biol. 1994;350:489-93

17. Bron AJ, de Paiva CS, Chauhan SK. et al. TFOS DEWS II Pathophysiology Report. Ocul Surf. 2017:15:438-510.

18. Baudouin C, Aragona P, Van Setten $G$, et al. Diagnosing the severity of dry eye: a clear and practical algorithm. Br J Ophthalmol. 2014;98:1168-76.

19. Kojima T, Dogru M, Kawashima M, et al. Advances in the diagnosis and treatment of dry eye. Prog Retin Eye Res. 2020;100842.

20. Buckley R. Assessment and management of dry eye disease Eye (Lond). 2018;32:200-3.

21. Sullivan $B D$, Whitmer $D$, Nichols $K K$, et al. An objective approach to dry eye disease severity. Invest Ophthalmol Vis Sci. 2010;51:6125-30.

22. Tomlinson A, Khanal S, Ramaesh, K et al. Tear film osmolarity: determination of a referent for dry eye diagnosis. Invest Ophthalmol Vis Sci. 2006;47:4309-15.

23. Huang JF, Zhang $\mathrm{Y}$, Rittenhouse $\mathrm{KD}$, et al. Evaluations of tear protein markers in dry eye disease: repeatability of measurement and correlation with disease. Invest Ophthalmol Vis Sci.
2012;53:4556-64.

24. Roy NS, Wei Y, Kuklinski E, Asbell PA. The growing need for validated biomarkers and endpoints for dry eye clinical research. Invest Ophthalmol Vis Sci. 2017;58:1-19.

25. Fong PY, Shih KC, Lam PY, et al. Role of tear film biomarkers in the diagnosis and management of dry eye disease. Taiwan 」 Ophthalmol. 2019:9:150-9.

26. Biomarkers Definitions Working Group. Biomarkers and surrogate endpoints: preferred definitions and conceptual framework. Clin Pharmacol Ther. 2001;69:89-95.

27. Hagan S, Martin E, Enríquez-de-Salamanca A. Tear fluid biomarkers in ocular and systemic disease: potential use for predictive, preventive and personalised medicine. EPMA 」. 2016;7(1):15

28. Lemp M, Sullivan B, Crews L. Biomarkers in dry eye disease. European Ophthalmic Review. 2012;6:157-63.

29. Baudouin C, Irke M, Messmer E, et al. Clinical impact of inflammation in dry eye disease: proceedings of the ODISSEY group meeting. Acta Ophthalmol. 2018;96:111-9.

30. Chiva A. Tear biomarkers in dry eye disease. European Ophthalmic Review. 2019:13:21-6.

31. Deschamps N, Baudouin C. Dry eye and biomarkers: present and future. Curr Ophthalmol Rep. 2013:1:65-74.

32. Versura P, Nanni P, Bavelloni A, et al. Tear proteomics in evaporative dry eye disease. Eye (Lond). 2010;24:1396-402.

33. Zhou L, Beuerman RW, Chan CM, et al. Identification of Tear Fluid Biomarkers in Dry Eye Syndrome Using iTRAQ Quantitative Proteomics. J Proteome Res. 2009;8:4889-905.

34. Suzuki M, Massingale M, Ye F, et al. Tear osmolarity as a biomarker for dry eye disease severity. Invest Ophthalmol Vis Sci. 2010;51:4557-61. 